Monatsschr Kinderheilkd 2009 • 157:1208-1208 DOI 10.1007/s00112-009-2099-2

๑) Springer-Verlag 2009
O. Bodamer ${ }^{1} \cdot$ R. Kerbl ${ }^{2}$

${ }^{1}$ Universitätsklinik für Kinder- und Jugendheilkunde, Paracelsus Medizinische Universität Salzburg

${ }^{2}$ Abteilung für Kinder und Jugendliche, LKH Leoben

\title{
Neugeborenenscreening im 21. Jahrhundert
}

Im Jahr 1961 entwickelte der US-amerikanische Mikrobiologe Dr. Robert Guthrie einen Screeningtest für auf Filterpapier getrocknetes Blut zum Nachweis erhöhter Phenylalaninkonzentrationen bei Phenylketonurie. Dieses ebenso einfache wie elegante Testverfahren legte den Grundstein für das Neugeborenenscreening, wie wir es heute als Teil der modernen Pädiatrie kennen. Bereits Dr. Guthrie erkannte, vor mehr als 40 Jahren, die Wichtigkeit einer frühzeitigen Diagnose und Therapie der Phenylketonurie innerhalb der ersten Lebenstage, um die später bei unbehandelten Kindern auftretende mentale Retardierung zu verhindern. Das Neugeborenenscreening ist somit zu einem wichtigen, weithin akzeptierten Bestandteil des öffentlichen Gesundheitswesens mit direkten und indirekten Vorteilen für die betroffenen Kindern und deren Familien geworden.

\section{Neugeborenenscreening-} programme sind weit verbreitet

Entsprechende Screeningprogramme gibt es mittlerweile - in unterschiedlichem Umfang - in allen Industrie- und vielen Schwellenländern. Trotz des stetigen Wandels der ethisch-moralischen, rechtlichen, ökonomischen und technologischen Rahmenbedingungen des Neugeborenenscreening per se sind die Unterschiede der Programme einzelner europäischer Länder nicht leicht begründbar.

Einen Überblick über den derzeitigen Stand und die verschiedenen Aspekte der Neugeborenenscreeningprogramme in Deutschland, der Schweiz und in Österreich gibt Dr. Zoltan Lukacs von der Universitätsklinik für Kinder- und Jugendme- dizin, Hamburg Eppendorf. Er geht auf die deutlichen Unterschiede im Hinblick auf die Anzahl der in Screeningprogrammen enthaltenen Krankheiten, die Größe der Screeninglabors sowie deren gesetzlichen Regulierung ein. Neben den angeborenen Stoffwechselstörungen spielen Endokrinopathien im Neugeborenenscreening eine wesentliche Rolle. Prof. Zabransky vom Institut für Pädiatrische Endokrinologie und Präventivmedizin (IPEP), Homburg, Saar, fasst den aktuellen Stand des Neugeborenenscreenings auf Hypothyreose und Adrenogenitales Syndrom zusammen und berichtet $u$. a. über die wichtigsten Pittfalls.

Frau Dr. Marina Stopsack vom Institut für klinische Chemie und Laboratoriumsdiagnostik des Universitätsklinikums Dresden geht in ihrem Beitrag auf das Pro und Kontra des Neugeborenenscreenings auf Mukoviszidose ein. Obwohl der Nutzen solcher Programme in der Zwischenzeit eindeutig belegt ist, sind in Europa lediglich in einigen wenigen Staaten (Großbritannien, Frankreich und Österreich) landesweite Programme etabliert. In anderen Ländern bestehen erfolgreiche, regionale Programme, so auch seit mehr als 12 Jahren in Ostsachsen. Die Schweiz hingegen steht kurz vor der Einführung eines entsprechenden Screeningprogramms.

\section{( Kosten-Nutzen-Abwägungen werden auch bei Screeningprogrammen immer wichtiger}

In Zeiten knapper Gesundheitsbudgets ist die Frage des Kosten-Nutzen-Verhält- nisses umso wichtiger. Frau Astrid Langer von der Fakultät für Betriebswirtschaft der Ludwig-Maximilians-Universität München beleuchtet diesen wichtigen Aspekt in ihrem Beitrag über das „Neugeborenenscreening im Spannungsfeld der Gesundheitsökonomie" anhand einer systematischen Auswertung von ökonomischen Evaluationsstudien zum Neugeborenenscreening mittels Tandemmassenspektrometrie.

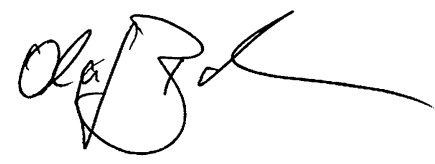

O. Bodamer

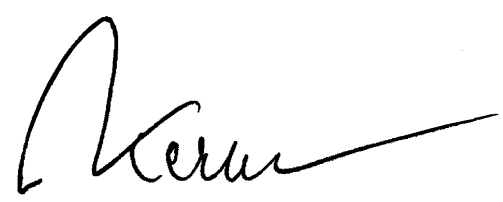

R. Kerbl

\section{Korrespondenzadresse}

Univ.-Doz. Dr. Dr. h.c. O. Bodamer FACMG

Universitätsklinik für Kinder- und Jugendheilkunde,

Paracelsus Medizinische Universität Salzburg Müllner Hauptstraße 48,

A-5020 Salzburg

Österreich

olaf.bodamer@pmu.ac.at 